\title{
Development of Thermosensitive Ophthalmic in situ Gels of Bimatoprost for Glaucoma Therapy
}

\author{
Sandeep Divate Sathyanarayana*, Narayana Charyulu Rompicherla, Anoop Narayanan \\ Vadakkepushpakath, Prashant Nayak
}

Department of Pharmaceutics, Nitte Gulabi Shetty Memorial Institute of Pharmaceutical Sciences, NITTE (Deemed to be University), Paneer, Deralakatte, Mangaluru, Karnataka, INDIA.

\begin{abstract}
Background: In recent years, ophthalmic in situ gels have gained wide importance for the sustained delivery of drugs into the eye by overcoming the demerits of conventional eye drops. Objectives: The present investigation was undertaken to formulate and evaluate Bimatoprost loaded thermosensitive ophthalmic in situ gels for providing prolonged drug release pattern with good patient acceptance. Methods: Bimatoprost thermosensitive ophthalmic in situ gels were prepared by the cold method using temperature dependent polymers, Poloxamer 188/poloxamer 407 in combination with HPMC K4M as viscosifier used in three different concentrations. The prepared in situ gels were evaluated for appearance, clarity, $\mathrm{pH}$, gelling capacity, gelation temperature, drug content and drug release study. The optimized batch of the formulation was subjected to drug release kinetics, ex vivo drug permeation, sterility, isotonicity, in vitro ocular irritancy test and short-term stability studies for 3 months. Results: From the drug release study, it was found that formulation; BT-5 had the highest drug release with higuchi release kinetic mechanism. The formulation (BT-5) was found to be sterile and the HET- CAM test confirmed that there was no ocular irritation and the formulation was stable for a period of 3 months without any significant changes in the evaluation parameters. Conclusion: Bimatoprost thermosensitive ophthalmic in situ gels can be a better alternative approach to provide sustained delivery of the drug by reducing the frequent drug instillation for the treatment of glaucoma.
\end{abstract}

Key words: Bimatoprost, Poloxamer 407, In situ gels, Viscosity, Isotonicity.

\section{INTRODUCTION}

Glaucoma is one of the complex eye disorders, which is characterized by a rise in Intra Ocular Pressure (IOP) than a normal eye can tolerate. Glaucoma is commonly known as a silent killer of vision loss and the second leading cause of blindness worldwide. Glaucoma is of two types- open angle and closed angle glaucoma. Open angle glaucoma is the chronic one affecting most of the people. ${ }^{1-4}$

Bimatoprost, which is a prostaglandin analogue, reduces the higher IOP by two mechanisms, pressure dependent and pressure independent. Recent studies in clinical trials prove that it is an effective drug remedy to control open angle glaucoma and has FDA scientific approval. ${ }^{5}$ Bimatoprost is now available as conventional eye drops, but eye drops have some demerits such as no sustained action, corneal elimination, less ocular contact time and lachrymal drainage.

In order to resolve the above said demerits of Bimatoprost eye drops, an attempt has been made to develop a stable, sustainable drug delivery system called ophthalmic in situ gels. ${ }^{6}$ These are the gelling liquids that convert the drug solutions into a gelling system, once the formulation is administered in to the eye. These undergo physico-chemical phase changes in the culde-sac of the eye and get convert into gels that retain in the corneal tissue with good contact time and release the drug for prolonged time periods for sustained action.
Submission Date: 28-11-2019; Revision Date: 06-02-2020; Accepted Date: 12-03-2020

DOI: 10.5530/ijper.54.2s.71 Correspondence: Mr. Sandeep Divate Sathyanarayana, M.Pharm, Assistant Professor, Department of Pharmaceutics, NGSM Institute of Pharmaceutical Sciences, NITTE (Deemed to be University), Paneer, Deralakatte,

Mangaluru-575018, Karnataka, INDIA.

Phone: +919036717475 E-mail: sandypharama@ gmail.com

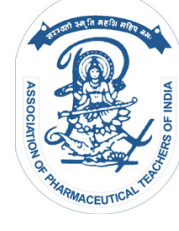

www.ijper.org 
Ophthalmic in situ gels can be prepared by three different approaches, change in $\mathrm{pH}$, change in the ionic system and change in temperature.

In situ gels prepared by change or rise in temperature are referred to as thermosensitive in situ gels, which undergo sol- gel transition by an increase in temperature from $20-25^{\circ} \mathrm{C}$ to $35-37^{\circ} \mathrm{C}$. The increase in temperature is characterized by cross linked polypropylenes called poloxamers which are hydrophilic in nature and give colorless transparent gel. ${ }^{8}$ Poloxamers are also called as pluronics which are responsible to form a micellar network to increase the temperature resulting into viscous transparent gelling liquids. Poloxamer 188 and poloxamer 407 were the two most commonly used thermosensitive polymers and their use in combination is found to be significant for effective in situ gelling systems. ${ }^{?}$

\section{MATERIALS AND METHODS}

Bimatoprost pure drug of the pharmaceutical grade was purchased from Dr. Pradeep Reddy's laboratory, Hyderabad. HPMC K4 M, Poloxamer 188 and Poloxamer 407 were procured from Hi media laboratory, Mumbai. Sodium chloride, Dibasic sodium phosphate, Sodium hydroxide and Benzalkonium chloride were procured from Lobachem suppliers, Mumbai.

\section{Methodology}

\section{Preparation of Bimatoprost loaded ophthalmic in situ gels}

\section{Selection of dose of Bimatoprost}

Dose of Bimatoprost in eye drops is $0.03 \%$ i.e each $\mathrm{ml}$ of formulation contains $0.3 \mathrm{mg}$ of $\operatorname{drug}(0.3 \mathrm{mg} / \mathrm{ml})$. A $9 \mathrm{mg}$ of Bimatoprost was calculated for preparing $30 \mathrm{ml}$ of in situ ophthalmic gels.

\section{Preparation of Bimatoprost ophthalmic in situ gels}

Thermosensitive ophthalmic in situ gels of Bimatoprost were prepared by the cold method. Poloxamer 188 was used in the concentration of $5 \%$ and poloxamer 407 was used in two different concentrations of 15 and $16 \%$ as temperature triggering polymers. HPMC K4 M was added in the different concentrations of $0.10,0.15$ and $0.20 \mathrm{~g}$ as viscosifying agent to enhance the viscosity. Dibasic sodium phosphate $(80 \mathrm{mg})$ was used as a buffering agent. Sodium chloride was used in the concentration of $0.9 \%$ for making the formulations isotonic and $0.01 \%$ of benzalkonium chloride was used as antimicrobial agent. ${ }^{10}$

All the above mentioned ingredients including Bimatoprost were added in $20 \mathrm{ml}$ of purified water and dissolved by a magnetic stirrer for sufficient time till the solutions become clear. To adjust $\mathrm{pH}$ in the ophthalmic range, $0.1 \mathrm{~N}$ sodium hydroxide was added dropwise as quantity sufficient and the final volume of in situ gels was made up to $30 \mathrm{ml}$ with purified water. All the formulated in situ gels were filtered through $0.22 \mu$ sterile syringe filters and transferred to $20 \mathrm{ml}$ sterile vials. The formulation composition of Bimatoprost loaded in situ gels is shown in Table 1.

\section{Evaluation of Bimatoprost thermosensitive ophthalmic in situ gels}

Appearance and flow nature: All the formulations were visually inspected for their general appearance and flow nature. ${ }^{11}$

Clarity: Clarity of all the formulations was donechecked under a fluorescent light against white and dark background. ${ }^{11}$

$\mathrm{pH}: \mathrm{pH}$ of the formulations was determined using a digital $\mathrm{pH}$ meter. An average of 3 trials was taken. ${ }^{12}$

\begin{tabular}{|c|c|c|c|c|c|c|}
\hline \multirow[t]{2}{*}{ Ingredients } & \multicolumn{6}{|c|}{ Formulation code } \\
\hline & BT-1 & BT-2 & BT-3 & BT-4 & BT-5 & BT-6 \\
\hline Bimatoprost (mg) & 9 & 9 & 9 & 9 & 9 & 9 \\
\hline HPMC K4M (g) & 0.10 & 0.15 & 0.20 & 0.10 & 0.15 & 0.20 \\
\hline Poloxamer $188(\%)$ & 5 & 5 & 5 & 5 & 5 & 5 \\
\hline Poloxamer 407 (\%) & 15 & 15 & 15 & 16 & 16 & 16 \\
\hline Sodium chloride (\%) & 0.9 & 0.9 & 0.9 & 0.9 & 0.9 & 0.9 \\
\hline Dibasic sodium phospahte (mg) & 80 & 80 & 80 & 80 & 80 & 80 \\
\hline Benzalkonium chloride (\%) & 0.01 & 0.01 & 0.01 & 0.01 & 0.01 & 0.01 \\
\hline $0.1 \mathrm{~N} \mathrm{NaoH}(\mathrm{ml})$ & $\begin{array}{c}\text { q.s to adjust } \\
\text { pH }\end{array}$ & $\begin{array}{c}\text { q.s to adjust } \\
\mathrm{pH}\end{array}$ & $\begin{array}{c}\text { q.s to adjust } \\
\mathrm{pH}\end{array}$ & $\begin{array}{c}\text { q.s to adjust } \\
\text { pH }\end{array}$ & $\begin{array}{c}\text { q.s to adjust } \\
\mathrm{pH}\end{array}$ & $\begin{array}{c}\text { q.s to adjust } \\
\text { pH }\end{array}$ \\
\hline Purified water q.s (ml) & 30 & 30 & 30 & 30 & 30 & 30 \\
\hline
\end{tabular}




\section{Gelling capacity}

Gelling capacity is one of the important criteria for ophthalmic in situ gels. It is the ability of the formulation to undergo sol to gel transition when the formulation is administered to cul de sac of eye. It was evaluated by placing a drop $(27 \mu \mathrm{l})$ of formulation in a vial containing $2 \mathrm{ml}$ of freshly prepared simulated tear fluid (composition- sodium chloride- $0.67 \mathrm{~g}$, sodium bicarbonate- $0.2 \mathrm{~g}$ and Calcium chloride $-0.008 \mathrm{~g}$ ) which was equilibriated at $37 \pm 2^{\circ} \mathrm{C}$ and the gelling capacity was visually evaluated by observing the time taken for gelation and time taken to dissolve the gel. ${ }^{13}$

The grading of the gelling capacity was shown below. Grading:

- No gelation

+ Gelation within few seconds and remained for a few minutes.

++ Gelation occurs immediately and remained for 6-8 hr. +++ Gelation occurs immediately and remained for an extended period.

++++ Stiff gels

\section{Viscosity measurement}

The viscosity of ocular in situ gels is important to determine the contact time between the drug and ocular tissue. The viscosity measurement was carried out by using Brookfield viscometer DVII + pro model. From the literature, it was evident that the formulation at $25 \pm 2^{\circ} \mathrm{C}$ should have a viscosity of $5-1000 \mathrm{cps}$ and at $37 \pm 2^{\circ} \mathrm{C}$ should have a viscosity of about $50-50,000$ cps. The samples were analyzed both before and after gelation using spindle no. 61 . The angular velocity of the spindle was increased from 5, 10, 20, 30, 50 and 100 and the viscosity of all the formulations was recorded. ${ }^{14}$

\section{Gelation temperature}

Transfer $10 \mathrm{ml}$ of the formulation in to a $50 \mathrm{ml}$ beaker and it is kept on a magnetic stirrer with a thermostatically controlled heater. The temperature of the stirrer was increased with the increment of $1^{\circ} \mathrm{C}$ and the temperature was checked using a thermometer. The rotation of the bead gradually becomes slow as gelation starts and the temperature at which the rotation of magnetic bead was stopped is considered as gelation temperature. ${ }^{15}$

\section{Drug Content Estimation}

$1 \mathrm{ml}$ of formulation equivalent to $100 \mathrm{mg}$ was taken and diluted with $100 \mathrm{ml}$ of simulated tear fluid to make a concentration of $10 \mu \mathrm{g} / \mathrm{ml}$. The final dilutions were made with simulated tear fluid according to the beer's range. The drug content was analyzed and estimated by taking the absorbance at $294 \mathrm{~nm}$ against the blank reagent using UV-Visible spectrophotometer. ${ }^{16}$

\section{In vitro drug release study}

In vitro drug release study was carried out by Franz diffusion cell consisting of donar and receptor compartments. The donar compartment was filled with $25 \mathrm{ml}$ of STF by placing a magnetic bead inside and the dialysis membrane which was soaked in STF for an overnight was mounted on the receptor compartment in such a way that dialysis membrane was in contact with STF in receptor compartment. Franz diffusion cell assembly containing STF was maintained at a temperature of $37 \pm 0.5^{\circ} \mathrm{C}$ and was rotated at a speed of $50 \mathrm{rpm}$. $1 \mathrm{ml}$ of in situ gel was placed on the dialysis membrane. Aliquots of $1 \mathrm{ml}$ were withdrawn at different time intervals of $0.5,1,2,3,4,5,6,7,8,9$ and $10 \mathrm{hrs}$ and $10 \mathrm{ml}$ of fresh STF was replaced. The withdrawn samples were diluted to $10 \mathrm{ml}$ with STF and were analyzed by UV spectrophotometer at $294 \mathrm{~nm}$. The percentage cumulative drug release $(\%$ CDR) was calculated. ${ }^{17}$

\section{Drug release kinetics}

The data obtained for in vitro release were fitted into the equation for the zero order, first order, higuchi and Koresmeyer- peppas release models. The best fitting model was selected on the basis of higher regression coefficient value $\left(R^{2}\right) .{ }^{18}$

\section{Ex vivo drug permeation study}

Ex vivo drug permeation study was carried out by using the modified Franz diffusion chamber and the goat corneal membrane was used for drug permeation study. The whole eyeball of the goat was procured from a slaughter house and carried to laboratory in the cold condition in normal saline maintained at $4^{\circ} \mathrm{C}$. From the eye ball, the cornea was carefully removed along with 5-6 $\mathrm{mm}$ of surrounding sclera tissue and the obtained cornea was washed with cold saline. Washed corneas were kept and soaked overnight in simulated tear fluid. The study was performed in the same manner as that of in vitro drug release procedure and the release study was done for a period of $12 \mathrm{hr}^{19}$

\section{Sterility test}

The sterility test was performed using soyabean casein digest media for fungi and fluid thioglycolate medium for bacteria. This test was performed according to the procedure of the direct inoculation method. The inoculated media were kept for incubation of 7 days and thereafter observed for the presence or absence 
of the microbial growth by comparing the optimized formulation with positive and negative controls. ${ }^{20}$

\section{Isotonicity test}

It was tested by mixing a few drops of blood with the optimized formulation and was observed under high resolution Biovis particle size analyzer microscope at 45X magnification. The procedure was repeated for marketed ophthalmic formulation for the comparison of RBCs. The RBCs were observed to check shrinkage, bulging of cells by comparing the shape of RBCs with optimized formulation and marketed formulation of Bimatoprost eye drops. ${ }^{21}$

\section{In vitro ocular irritancy evaluation by HET-CAM (Hen's egg test choriallantonic membrane) test}

Since the Draize Rabbit test has been banned, an OECD recommended in vitro ocular irritation test was conducted known as the HET-CAM test. In this test, freshly collected white leghorn chicken eggs (not older than 7 days) weighing between 50-60 g were used and those with physical damage, cracks were rejected. Three groups were made each containing 3 eggs.

Negative control: Here the eggs were treated with $0.3 \mathrm{ml}$ of $0.9 \% \mathrm{Nacl}$ as a standard.

Test group: In this group eggs were tested with $0.3 \mathrm{ml}$ of the optimized formulation.

Positive control: In this, eggs were treated with $0.3 \mathrm{ml}$ of $1 \%$ SDS (Sodium dodecyl sulfate) as an irritant for comparison with negative control and test.

Procedure: The eggs were kept on a tray and placed in an incubator maintained at a temperature of $37 \pm 0.5^{\circ} \mathrm{C}$ and relative humidity of $58 \pm 2^{\circ} \mathrm{C}$. Manually the eggs were rotated 5 times per day for about 8 days. Candle the eggs on the $8^{\text {th }}$ day of incubation to confirm the embryo growth. On confirmation, eggs were replaced into the incubator without rotation by keeping the large end upward and kept for one complete day. On the $9^{\text {th }}$ day; mark the air cell on top and make a hole on the air sac of egg shell without injuring the membrane. All the eggs were treated with respective solutions and observed for the signs of hemorrhage, coagulation and lysis of blood vessels for a time period of $300 \mathrm{sec}(5$ $\min )^{22,23}$

The irritation score (IS) formula is given below followed by irritation score value with inference in Table 2 .

$$
\mathrm{IS}=\frac{(301-\mathrm{H})}{300} \times 5+\frac{(301-\mathrm{L})}{300} \times 7+\frac{(301-\mathrm{C})}{300} \times 9
$$

Where, H- Hemorrhage

$$
\text { L- Lysis of blood vessels }
$$$$
\text { C- Coagulation }
$$

Table 2: Irritation score value with inference for HET-CAM Test.

\begin{tabular}{|c|c|}
\hline Irritation Score & Inference \\
\hline $0-0.9$ & No irritation \\
\hline $1-4.9$ & Weak irritation \\
\hline $5-8.9$ & Moderate irritation \\
\hline $9-21$ & Severe irritation \\
\hline
\end{tabular}

\section{Short term Stability studies for optimized} formulation

The ICH has framed certain conditions and procedures to conduct short term stability studies to predict the physical and chemical stability of drug products. For ophthalmic preparations, the study was conducted for a time period of 3 months. The optimized formulation was stored in a glass vial and kept in the stability chamber at room temperature of $25 \pm 2^{\circ} \mathrm{C}$ and $60 \pm 5 \%$ Relative Humidity $(\mathrm{RH})$ and at the accelerated temperature of $40 \pm 2^{\circ} \mathrm{C}$ and $65 \pm 5 \%$ Relative Humidity $(\mathrm{RH})$. The parameters namely appearance, clarity, $\mathrm{pH}$ and drug content were evaluated for 1,2 and 3 months to verify the stability of optimized formulation..$^{24,25}$

\section{RESULTS AND DISCUSSION}

\section{FTIR Spectroscopy}

From the interpretation of the FTIR study, it was confirmed that the drug Bimatoprost was compatible with the polymers used for the formulation of ophthalmic in situ gels and there was no additional peak in the spectra's, confirming that there was no chemical interaction between the drug and polymers used.

The FTIR spectra of Bimatoprost, physical mixture of Bimatoprost with poloxamer 188 and poloxamer 407 are represented in Figure 1.
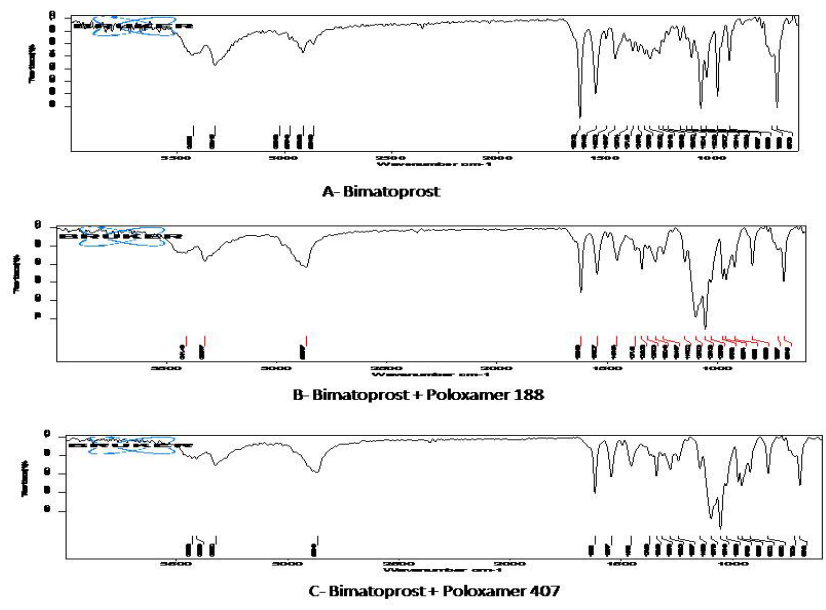

Figure 1: IR Spectrum of Pure Bimatoprost (A), Bimatoprost + Poloxamer 188 (B) and Bimatoprost + Poloxamer 407 (C). 


\section{Appearance}

All the six formulations were found to be transparent clear liquids with free flowing nature. The results are shown in Table 3.

\section{Clarity}

All the prepared Bimatoprost ophthalmic in situ gels were found to be clear without the presence of any particles and particulate matter. The results are shown in Table 3.

\section{$\mathrm{pH}$}

The $\mathrm{pH}$ of all the formulations was in the range of 6.8-7.3 and found to be within the physiological range of the human eye. The results are shown in Table 3.

\section{Gelling capacity}

All the formulations showed immediate gelation and remained for an extended period of time. The formulations with $15 \%$ of poloxamer 407 showed the gelling capacity for about $8 \mathrm{hr}$ and the remaining three formulations with $16 \%$ of poloxamer 407 showed the gelling capacity for about $10 \mathrm{hr}$. The increase in gelling capacity was due to the increase in the concentration of poloxamer from $15-16 \%$ where the gelation time is exceeded due to the increased formation of micelles from polypropylene polymers. The results of the gelling capacity are shown in Table 3.

\section{Rheological Study}

Based on the results, it was found that formulations showed the viscosity ranging from 7.65 to $82.24 \mathrm{cps}$ at $25 \pm 2^{\circ} \mathrm{C}$ before gelation and $238.16-2335.52 \mathrm{cps}$ at $37 \pm 2^{\circ} \mathrm{C}$ after gelation. The increase in viscosity was dependent on the concentration of HPMC K4M. The viscosity of the formulation was gradually increased with increase in the concentration of HPMC K4M. The viscosity of formulations was found to increasing with a decrease in rpm and the rheological graphs showed the Psuedoplastic flow nature of liquids. These shear rate properties of the in-situ gels can uniformly distribute the drug on the ocular surface and increase the ocular contact time for providing prolonged drug release. The results of viscosity for the in-situ gels before and after gelation were depicted in Figures 2 and 3.
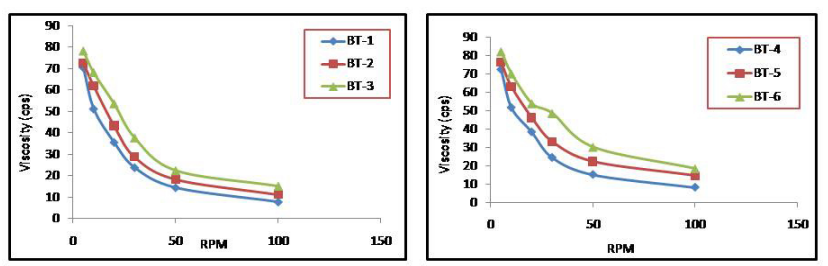

Figure 2: Viscosity of Bimatoprost in situ gels before gelation.
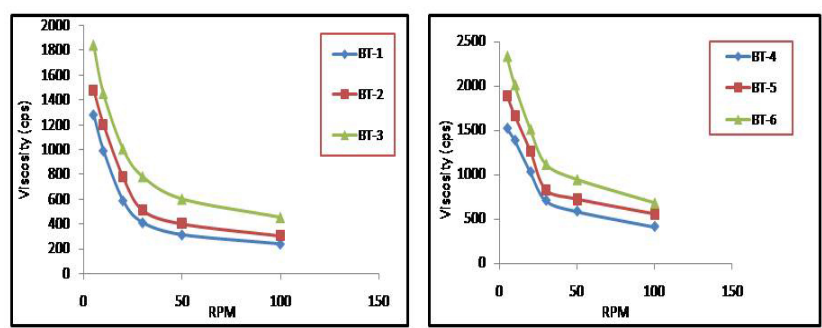

Figure 3: Viscosity of Bimatoprost in situ gels after gelation.

\section{Gelation temperature}

The gelation temperature of all the six formulations was found to be $36.8-37.8^{\circ} \mathrm{C}$. The formulations with $5 \%$ and $15 \%$ of poloxamer 188 and poloxamer 407 showed gelation temperature of 36.4 to $36.3^{\circ} \mathrm{C}$ whereas formulation BT-2 shown the temperature of $37.3^{\circ} \mathrm{C}$ which may be due to the optimum amount of HPMC $\mathrm{K} 4 \mathrm{M}(0.15 \mathrm{~g})$ the formulation was not much viscous to influence the gelling capacity by micelle formation. A similar observation was seen in the formulation BT-5 which showed a higher temperature of $37.5^{\circ} \mathrm{C}$ in comparison to the remaining two formulations, BT-4 and BT- 6 which showed a temperature of 37.2 and $36.6^{\circ} \mathrm{C}$. From the study, it was revealed that gelation temperature was increased with higher gelling capacity and at the same time the higher concentration of HPMC K4M which increases the viscosity of the in situ gels and reduces the gelation temperature by reducing the formation of the micelles. The results for the gelation temperature of the formulations are shown in Table 3.

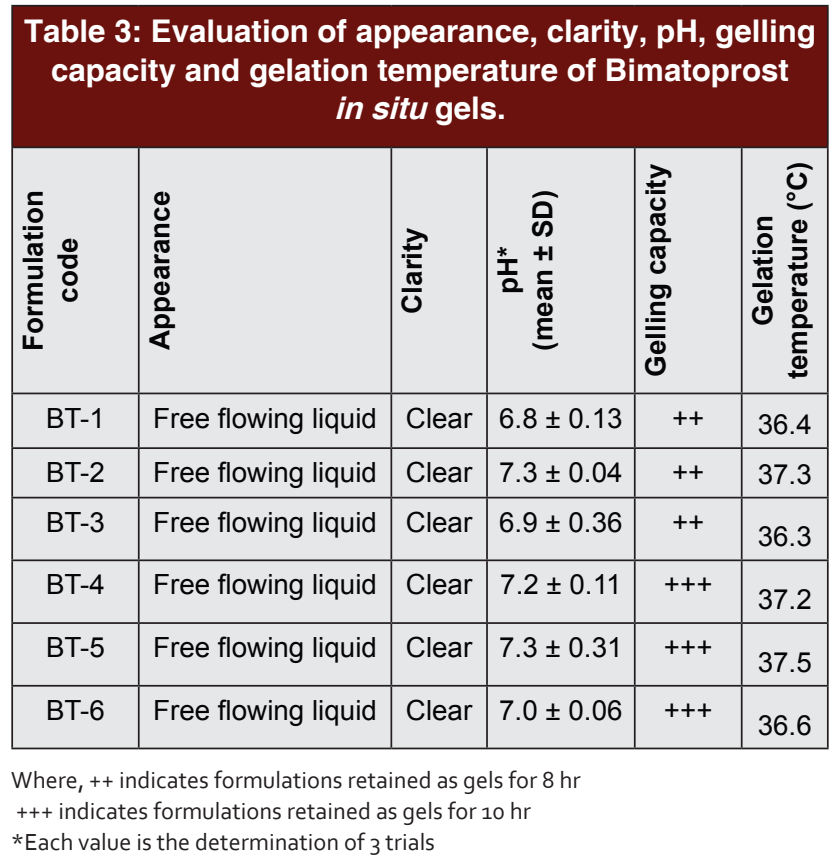




\section{Drug content estimation}

The drug content of all the six formulations of Bimatoprost in situ gels was estimated by UV spectroscopy measuring the absorbance at $294 \mathrm{~nm}$ against blank and the drug content of all the formulations was in the range of $92.18-95.63 \%$.

\section{In vitro drug release study}

The formulations BT-1, BT-2 and BT-3 containing 5\% poloxamer 188 and $16 \%$ poloxamer 407 showed the drug release of $75.38-81.26 \%$ up to $8 \mathrm{hr}$ since the gelling capacity of these formulations was retained for $8 \mathrm{hr}$ and the formulations BT-4, BT-5 and BT-6 containing $5 \%$ poloxomer 188 and $17 \%$ poloxomer 407 showed the release of drug from $80.37-86.63 \%$ upto $10 \mathrm{hrs}$ since the gelling capacity of these formulations was retained for an extended period of $10 \mathrm{hrs}$. It has been observed that drug release of all the formulations was increased with respect to the concentration of HPMC K4M in the concentration range of $0.10 \mathrm{~g}-0.15$ $\mathrm{g}$ and the drug release was reduced with increased concentration of $0.2 \mathrm{~g}$ of HPMC K4M because higher the viscosity of formulation lesser will be the permeation through the membrane and finally the release amount of the drug also will be lesser. Based on the results, it was confirmed that among all, formulation BT-5 shown the highest drug release of $86.63 \%$ up to $10 \mathrm{hr}$ and emerged as best.

This might be due to an increase in the concentration of poloxamer $407(17 \%)$ and the optimum concentration of HPMC K4M $(0.15 \mathrm{~g})$ as a viscosifying agent. Hence formulation BT-5 was chosen as the optimized and was subjected to other evaluation parameters such as drug release kinetics study, ex vivo drug permeation study, sterility test and Isotonicity test. The drug release profile of formulated in situ gels was depicted in Figure 4.

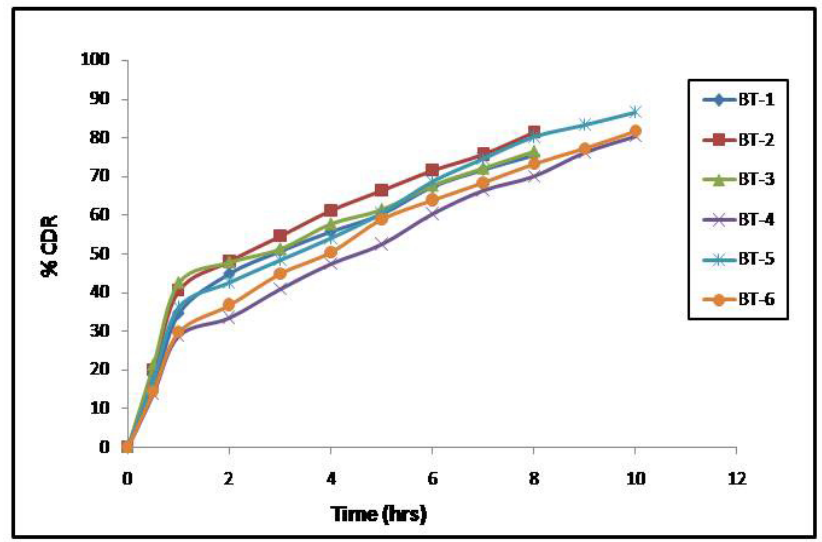

Figure 4: Drug release profile of Bimatoprost in situ gels.

\section{Drug release kinetics}

Based on the results, it was observed that higuchi release kinetics showed the higher regression $\left(R^{2}\right)$ of 0.988 indicating that the release of drug follows swelling of the polymeric system followed by diffusion system for sustaining the drug release. The higuchi release kinetics profile for the optimized formulation, BT-5 was represented in Figure 5.

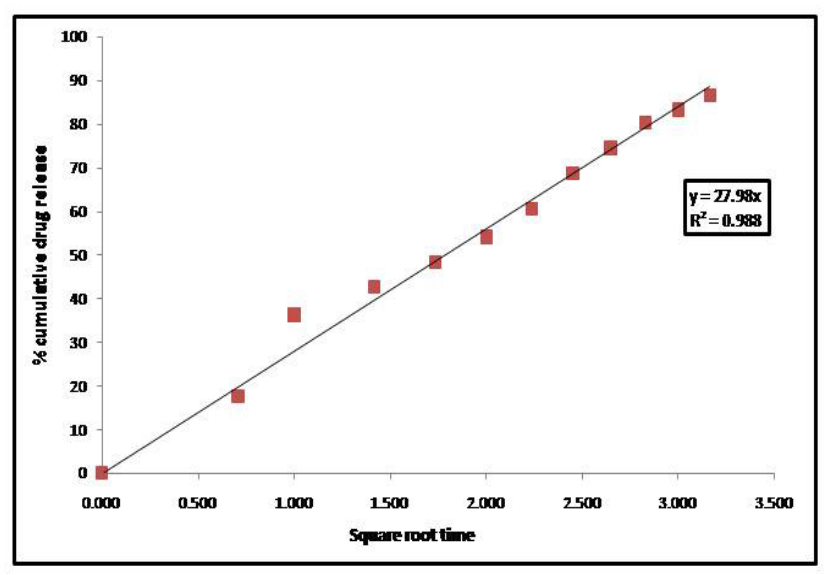

Figure 5: Higuchi drug release kinetics for BT-5.

\section{Ex vivo drug permeation study}

The drug permeation for the BT-5 in situ gel was found to be $67.45 \%$ upto $12 \mathrm{hr}$. The slow release of the drug may be because of the fact that cornea is made up of many layers like epithelium, stroma and endothelium which are highly lipophilic in comparison with a dialysis membrane and the formulation may take more time to diffuse through these layers for the release of the drug. The ex vivo drug permeation along with in vitro release comparison for BT-5 is shown in Figure 6.

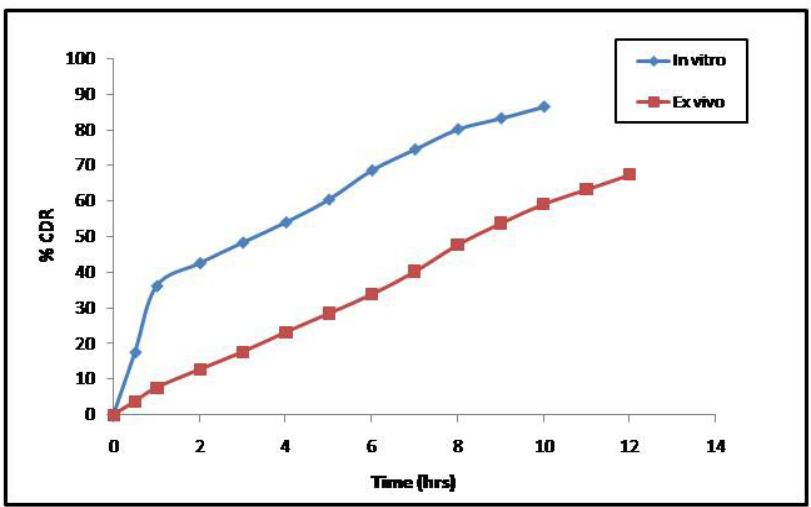

Figure 6: Comparison of in vitro and ex vivo drug release of BT-5. 


\section{Sterility test}

In positive control (Growth promotion test) it was observed that both medias (Fluid thioglycolate and soyabean casein digest agar) promoted the growth of microorganisms after the incubation of 7 days indicated by the appearance of turbidity, whereas there was no microbial growth in negative control and in the optimized formulation (BT-5), confirming that the formulation was found to be sterile in nature.

\section{Isotonicity test}

The Isotonicity test revealed that there was no shrinkage or bulging of RBCs with optimized formulation (BT-5) when compared with the marketed formulation. It was confirmed that the formulation was found to be isotonic with RBCs.

\section{In vitro ocular irritancy test by HET-CAM Method for BT-5}

The eggs of positive control treated with 1\% SDS showed irritation mean score of 14.07 indicating severe irritation with blood vessels lysis and hemorrhage in the chick embryo, the negative control group treated with $0.9 \% \mathrm{Nacl}$ showed mean score of 0.04 and the mean score of optimized formulation was 0.05 which confirms that there was no irritation in the chick embryo and the formulation was found to be non-irritant and non-toxic when compared with positive and negative control groups.

The results of mean irritation score for the negative control, positive control and optimized formulation were discussed in Table 4 and the HET-CAM test images are depicted in Figure 7.

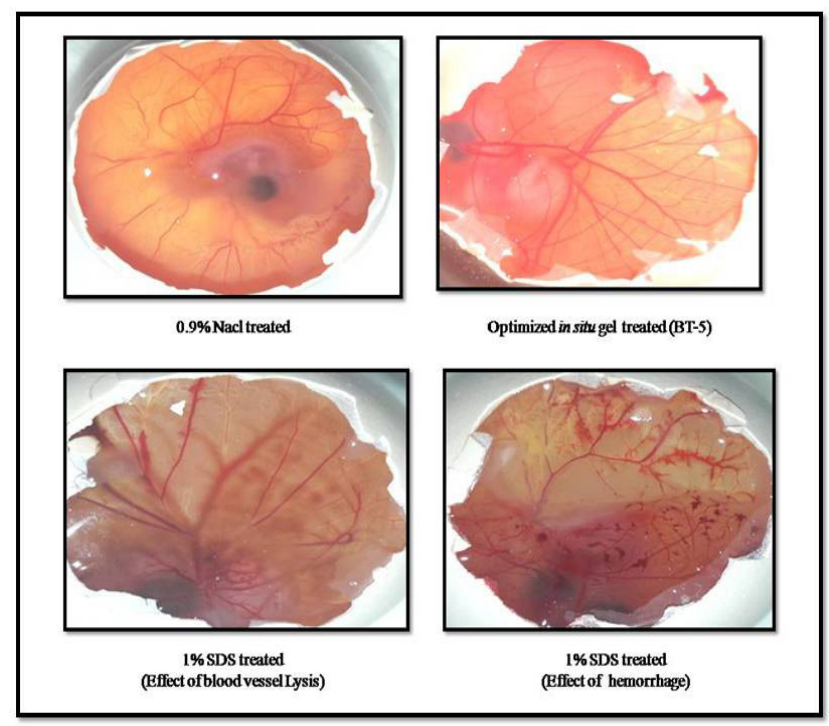

Figure 7: HET- CAM test for optimized formulation (BT-5).

\begin{tabular}{|c|c|c|}
\hline \multicolumn{3}{|c|}{ Table 4: In vitro ocular irritancy for BT-5 by } \\
HET-CAM test. & Inference \\
\hline Test compound & $\begin{array}{c}\text { Mean Irritation } \\
\text { Score }\end{array}$ & No irritation \\
\hline $\begin{array}{c}0.9 \% \text { Nacl (Negative } \\
\text { control) }\end{array}$ & 0.04 & Severe irritation \\
\hline $\begin{array}{c}1 \% \text { SDS (Positive } \\
\text { control) }\end{array}$ & 14.07 & No irritation \\
\hline $\begin{array}{c}\text { Optimized formulation } \\
\text { (BT-5) }\end{array}$ & 0.05 & \\
\hline
\end{tabular}

\section{Stability studies for BT-5 formulation}

The optimized formulation of Bimatoprost (BT-5) was evaluated for appearance, clarity, $\mathrm{pH}$ and drug content for their stability for a short term of 3 months. From the results, it was found that optimized formulations did not show any significant changes in the parameters evaluated and the formulation was found to be stable for a period of 3 months. The results of stability studies for optimized formulations were discussed in Table 5.

Table 5: Results of appearance, clarity, $\mathrm{pH}$ and drug content for BT-5 at $25 \pm 2^{\circ} \mathrm{C} / 60 \pm 5 \% \mathrm{RH}$ and at $40 \pm 2^{\circ} \mathrm{C} / 65 \pm 5 \%$ RH.

\begin{tabular}{|c|c|c|c|c|}
\multicolumn{5}{|c|}{ Stability studies at $25 \pm 2^{\circ} \mathrm{C} / 60 \pm 5 \%$ RH for optimized } \\
Bimatoprost in situ gel (BT-5)
\end{tabular}

\section{CONCLUSION}

The current research study was an attempt to formulate thermosensitive ophthalmic in situ gels for Bimatoprost for the management of glaucoma. Bimatoprost ophthalmic in situ gels were prepared by the cold method using two temperature dependent polymers poloxamer 188 and poloxamer 407, HPMC K4M was used in three different concentration to enhance the viscosity of formulations. 
Over all six formulations were made by varying concentrations of poloxamer 407 and HPMC K4M. Drug release study showed that in situ gels prepared with $16 \%$ poloxamer 407 were remained for release of drug up to $10 \mathrm{hr}$ and formulation BT-5 was optimized as best among all showed highest drug release with higuchi release kinetics and was found to be strile, isotonic and non-irritant in nature. The optimized formulation (BT-5) was stable for a period of 3 months without any changes in the evaluation parameters. Hence from the results of the present investigation, it can be concluded that Bimatoprost thermosensitive ophthalmic in situ gels can be successfully be formulated as an effective and better alternative drug delivery approach for the glaucoma.

\section{ACKNOWLEDGEMENT}

The authors would like to thank and acknowledge the authorities of NGSM Institute of Pharmaceutical Sciences, NITTE (Deemed to be University) for providing lab facilities to carry out this project work.

\section{CONFLICT OF INTEREST}

The authors declare that there was no conflict of interest.

\section{ABBREVIATIONS}

IOP: Intra ocular pressure; FDA: Food and drug administration; STF: Simulated tear fluid, q.s: Quantity sufficient; HET-CAM: Hen's egg test choriallantonic membrane; mg: Milligram; ml: Millilitre; $\mu \mathrm{g}$ : Microgram; min: Minutes; hr: Hours; ${ }^{\circ} \mathbf{C}$ : Degree Celsius; RH: Relative humidity; Nacl: Sodium chloride; SDS: Sodium dodecyl sulfate; RBCs: Red blood cells; nm: Nanometer; g: Gram; rpm: Revolutions per minute; cps: Centipoise; FTIR: Fourier transform infra red; IS: Irritation score; OECD: Organization for economic co-operation and development

\section{REFERENCES}

1. Natalie S, Gustav S, Selente B. Glaucoma: A brief review. S Afr Pharm J. 2018;82(5):18-22.

2. Paul S, Mondol R, Ranjit S, Maiti S. Anti Glaucomatic Niosomal System: Recent Trend in Ocular Drug Delivery. Int J Pharm Pharm Sci. 2010;2(2):15-8.
3. Sandeep DS, Narayana CR, Anoop NV. Smart in situ gels for glaucoma: An overview. Int J Pharm Sci Rev Res. 2018;50(1):94-100.

4. Dubey A, Prabhu P. Ocular drug delivery systems for treatment of glaucoma. Int J Pharm Sci Nanotech. 2014;7(2):2412-22.

5. Robert JN, Melissa LE. An update on bimatoprost in glaucoma therapy. Therapy. 2004;1(1):31-41.

6. Jothi M, Harikumar SL, Geeta A. In -situ ophthalmic gels for the treatment of eye diseases. Int J Pharm Sci Res. 2012;3(7):1891-904.

7. Ahmed VA, Goli D. Xyloglucan based in situ gel: Formulation Development and evaluation of in situ ophthalmic gel of Brimonidine tartarate. Indo Global J Pharm Sci. 2018;8(3):92-103.

8. Priyanka DD, Shahi SR, Swati SV. Ophthalmic in situ gel: A review. Eur J Pharm Med J. 2016;3(6):205-15.

9. Ramyadevi D, Sandhya P, Vedhahari BN. Poloxamer: A novel functional molecule for drug delivey and gene therapy. J Pharm Sci Res. 2013;5(8):159-65.

10. Rajeshwari NP, Rachana SK. In situ gelling system: Novel approach for ophthalmic drug delivery. World J Pharm Pharm Sci. 2014;3(7):423-40.

11. Ramanjit S, Seema S, Gurpreet S, Banerjee A. In situ gels- A new trends in ophthalmic drug delivery systems. IJPSR. 2015;6(5):886-90.

12. Himanshu G, Aqil M, Khar RK, Ali A, Mittal G. An alternative in situ gel formation of levofloxacin eye drops for prolong ocular retention. J Pharm Bioall Sci. 2015;7(1):9-14.

13. Shradha M, Gowda DV, Gupta NV, Bhavya MV. Fabrication, characterization and evaluation of in situ gel for the treatment of conjunctivitis. J Pharm Sci Res. 2018;10(5):1220-2.

14. Jingfen $S$, Zhengshen $Z$. A novel ocular delivery of brinzolamide based on gellan gum: In vitro and in vivo evaluation. Drug Design Dev Therapy. 2018;12:383-9.

15. Katpale AT, Shinde A. Formulation and evaluation of thermoreversible in situ ocular gel of clonidine hydrochloride for glaucoma. Pharmacophore. 2015;6(5):220-32.

16. Bassi B, Chandan S, Angshu B. Formulation and evaluation of ocular in situ gels using lincomycin hydrochloride. Int $\mathrm{J}$ Recent Adv Pharm Res. 2015;5(3):43-70.

17. Harish DB, Savkare A, Ritesh K, Derle D. Development of poloxamer based thermosensitive in situ ocular gel of betaxolol hydrochloride. Int J Pharm Pharm Sci. 2015;7(6):287-91.

18. Sherif AY, Gamal MM, Alanazi FK. Novel in situ gel for intravesicle administration of ketorolac. Saudi Pharm J. 2018;26(6):845-51.

19. Ankit A, Prakash G, Chitti R, Basavaraj MD. Preparation of gellan gum and chitosan based in situ gel of timolol maleate for ophthalmic drug delivery and evaluation of physcicochemical properties and drug release profile. Acta Scient Pharm Sci. 2019;3(2):68-78.

20. Sravankumar A, Rajkumar J, Vinaya J. Formulation and evaluation of flurbiprofen ocular in situ gel. Int J Pharm Sci Res. 2018;9(5):1851-6.

21. Nazia K, Aqil M, Syed SI, Ali A. Development and evaluation of a novel in situ gel of sparfloxacin for sustained ocular drug delivery: In vitro and ex vivo characterization. Pharm Dev Technology. 2015;20(6):662-9.

22. Vinardell MP, Mitjans M. Alternative methods for eye and skin irritation tests: An overview. J Pharm Sci. 2008;97(1):46-59.

23. HET-CAM test, Methods in molecular biology, ECVAM DB-ALM: INVITOXX protocol. 1995.

24. Stability testing of new drug substances and products. ICH Q1A. ICH harmonized triplicate guidelines of EMEA. 2003;1-20.

25. European Medicines Agency. Guidance on stability testing of new drug substances and products. EMEA. 2006. Available from: https://www.ema. europa.eu/en/documents/scientific-guideline/ich-q-1-r2-stability-testing-newdrug-substances-products-step-5_en.pdf. 


\section{PICTORIAL ABSTRACT}

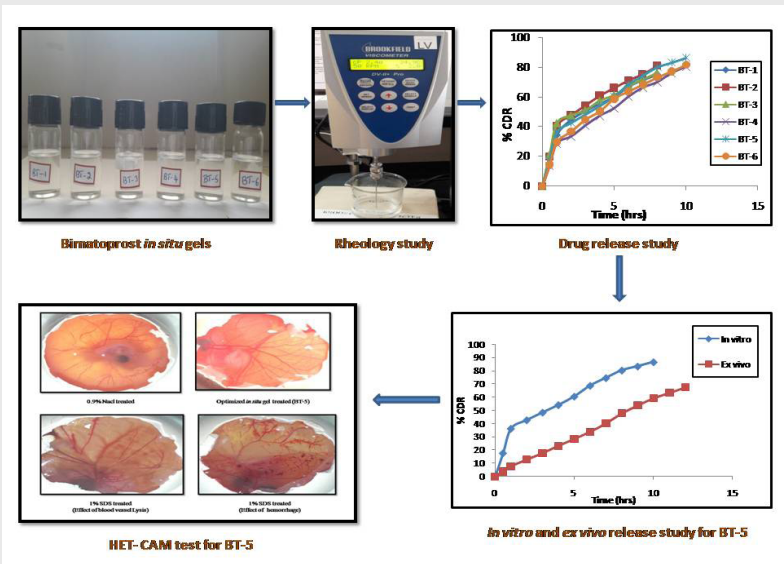

\section{SUMMARY}

- The present work was an attempt to prepare thermoensitive ophthalmic in situ gels for Bimatoprost for the glaucoma therapy. Poloxamer188/407 were used in combination as temperature triggering polymers and HPMC $\mathrm{K} 4 \mathrm{M}$ was used as viscosity enhancer.

- The formulated Bimatoprost ophthalmic in situ gels were evaluated for appearance, clarity, $\mathrm{pH}$, gelling capacity, viscosity, gelation temperature, in vitro drug release, ex vivo drug permeation, sterility test, isotonicity test and in vitro ocular irritancy test.

- Bimatoprost thermosenstive ophthalmic in situ gel showed sustained drug release with isotonic and non-irritant properties and the formulation was found to be stable for a period of three months.

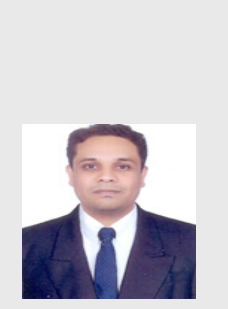

\section{About Authors}

Mr. Sandeep Divate $\mathbf{S}$ is currently working as Assistant Professor in the Department of Pharmaceutics at NGSM Institute of Pharmaceutical Sciences, Nitte (Deemed to be University) Mangalore. He has 2 years of industrial experience and 6 years of academic experience. His area of research includes ocular drug delivery systems, topical formulations and solid lipid nanoparticles.

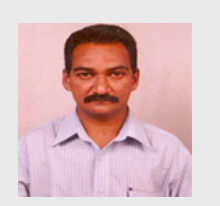

Dr. Narayana Charyulu R is presently serving as Vice principal and Head of the Pharmaceutics Department at NGSM Institute of Pharmaceutical Sciences, Nitte (Deemed to be University) Mangalore. He has 29 years of teaching experience and has published several national and international research papers.

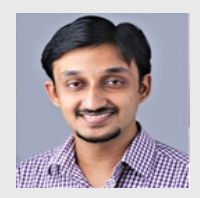

Dr. Anoop Narayanan $\mathbf{V}$ is presently working as Assistant Professor in the Pharmaceutics Department at NGSM Institute of Pharmaceutical Sciences, Nitte (Deemed to be University) Mangalore. His area of research includes silver and gold nanoparticles for cancer therapy.

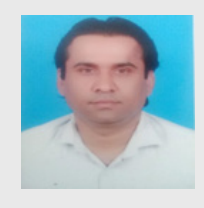

Mr. Prashant Nayak is currently working as Assistant Professor in the Pharmaceutics Department at NGSM Institute of Pharmaceutical Sciences, Nitte (Deemed to be University) Mangalore. His area of research includes drug design, molecular biology and nanoformulations.

Cite this article: Divate SS, Charyulu NR, Narayan AV, Nayak P. Development of Thermosensitive Ophthalmic in situ Gels of Bimatoprost for Glaucoma Therapy. Indian J of Pharmaceutical Education and Research. 2020;54(2s):s154-s162. 